\title{
Anisotropic Janus Catalysts for Spatially Controlled Chemical Reactions
}

\author{
Weipeng Lv, Kyung Jin Lee, Jinjing Li, Tae-Hong Park, Sangyeul Hwang, \\ A. John Hart, Fengbao Zhang, and Joerg Lahann*
}

\begin{abstract}
Anisotropic micro-/nanoparticles have been of particular interest due to their potential applications as smart drug carriers, self-assembled hierarchical structures, and biomimetic colloidal building blocks. ${ }^{[1-6]}$ In the last decade, several scientific advances led to the preparation of a diverse range of anisotropic particles including particles with different shapes, patchy particles, and compartmentalized particles. ${ }^{[1-6]}$ An emerging bulk of research has been focused on the preparation of multicompartmentalized polymer micro- and nanoparticles. ${ }^{[6-11]}$ Compared to particles with anisotropic distribution of surface patches, multicompartmentalized particles may have several additional properties, such as anisotropic degradation or spatially controlled swelling/deswelling within the same particle. ${ }^{[6-8,12,13]}$ Some of the potentially most promising applications may involve anisotropic catalysts that can induce spatially separated chemical reactions. ${ }^{[12]}$ As described by Crossley et al., Janus catalysts could control chemical reactions at the interface of oil and water, which enhances reaction activity relative to homogenous catalysts, while facilitating simple catalyst recovery. ${ }^{[12]}$ Anisotropic catalyst particles may
\end{abstract}

W. Lv, ${ }^{[+]}$Dr. K. J. Lee, ${ }^{[+]}$Dr. T.-H. Park, ${ }^{[++]}$Dr. S. Hwang,

Prof. J. Lahann

Department of Chemical Engineering

University of Michigan

Ann Arbor, MI 48109, USA

E-mail: Lahann@umich.edu

W. Lv, ${ }^{[+]}$Prof. F. Zhang

School of Chemical Engineering and Technology

Tianjin University

Tianjin 300072, China

J. Li, Prof. A. J. Hart

Department of Mechanical Engineering

University of Michigan

Ann Arbor, MI 48109, USA

Prof. J. Lahann

Departments of Mechanical Engineering

Materials Science and Engineering

Macromolecular Science and Engineering

and Biomedical Engineering

University of Michigan

Ann Arbor, MI 48109, USA

[+] W. L. and K. J. L. contributed equally to this work.

[++] Current address: Nuclear Chemistry Research Division, Korea

Atomic Energy Research Institute (KAERI), Daejeon, 305-353, Korea

DOI: $10.1002 / \mathrm{smll} .201200192$ also enable self-propellant microengines that can be fueled by bubbles generated by spatially controlled catalytic reactions. ${ }^{[14,15]}$ Posner's groups presented rapid fabrication of bimetallic spherical motors by spatial coating of metal nanoparticles on polystyrene microbeads, showing effective swimming microengines fueled by controlled hydrogen peroxide decomposition on one side. ${ }^{[15 a]}$ More recently, Baraban et al. reported catalytic Janus motors with controlled deposition of magnetic nanoparticles, where the motion of Janus motors can be controlled by external magnetic fields. ${ }^{[15 b]}$ Beyond Janus architectures, multicompartmental catalysts composed of several distinct pockets of reactivity may enable cascade reactions, mimicking the complex chemical flows in biological cells. ${ }^{[7,13]}$

Major challenges related to multicompartmental catalytic applications are linked to the small dimensions required for Janus catalysts and the lack of thermal stability of polymers typically used to prepare multicompartmental particles. ${ }^{[4,7,12]}$ A potential solution may be provided by bimetallic catalyst nanoparticles, but their preparation has been notoriously challenging and tedious. ${ }^{[16,17]}$ An alternative pathway may include the preparation of multicompartmental catalytic nanoparticles displaying different catalysts in each compartment. For this type of catalytic supporter particles, inorganic supporter materials, such as silica and titania, are preferential over polymeric materials, mainly because of the higher thermal and chemical stability.

Electrospraying provides a simple, yet versatile and scalable platform for fabricating nanoparticles with controlled size, shape, and internal architecture. ${ }^{[18-24]}$ Employment of more complex needle configurations with multiple parallel flows (so-called electrohydrodynamic (EHD) co-jetting) has been instrumental in extending EHD co-jetting towards the production of multicompartmental polymer particles with unique internal architectures. ${ }^{[21-24]}$ However, the inorganic microstructures produced by electrospinning are mostly dependent on a sol-gel based methods with waterborne polymers, such as poly(vinyl alcohol) and poly(vinyl pyrolidone). ${ }^{[25-27]}$

Herein, we describe a novel process for producing multicompartmental inorganic particles using EHD co-jetting of composite solutions followed by thermal calcination at $500{ }^{\circ} \mathrm{C}$. In this approach, the role of the degradable organic polymer is that of a template, which ensures homogeneous particle formation during side-by-side EHD co-jetting. The 
green and blue compartments suggests a bicompartmental architecture of the initial Janus particles. ${ }^{[21-24]}$ In addition, electron microscopy indicates a near-perfect spherical shape of the particles. This is an important aspect of the synthesis approach because the previously reported shapes of inorganic materials produced by electrohydrodynamic jetting have been limited to fibrillar structures. ${ }^{[24-26]}$ This is because the previously employed precursor solutions already undergo sol-gel reactions during the jetting process. In the case of jetting of reactive sol-gel systems, continuous gelation during jetting increases the viscosity of the jetting solutions. Therefore, the solution viscosities are no longer dependent on the initial polymer concentrations as the sol-gel reactions precede. As the solution

Scheme 1. A) Schematic illustration of the electrohydrodynamic co-jetting process used to produce bicompartmental organic/inorganic composite particles. B) Schematic diagram for preparation of the Janus catalysts for spatially confined growth of carbon nanotubes on one hemisphere of the particles.

sacrificial polymer is then removed under the conditions of the subsequent calcination reaction. Following this strategy, catalytically active additives, such as magnetic nanocrystals can be spatially confined to one side of the Janus catalysts.

The typical experimental set-up for EHD co-jetting of inorganic composite materials is displayed in Scheme 1A. Two separate polymer solutions were prepared by mixing poly(DLlactide-co-glicolide) (PLGA; $6.0 \mathrm{w} / \mathrm{V} \%$ ) solution with titanium (IV) butoxide (TTBO, titania precursor) $(9.0 \mathrm{~V} / \mathrm{V} \%)$ in anhydrous chloroformdimethylformamide (DMF; 97/3 V/V). Magnetic nanocrystals with an average diameter of $10 \mathrm{~nm}$ were added to one of the two jetting solutions. In order to visualize the particles by confocal laser scanning microscopy (CLSM), trace amounts of blue (poly[ $(m$-phenylenevinylene)-alt-(2,5dibutoxy-p-phenylenevinylene)] (MEHPV), $\sim 0.05 \mathrm{w} / \mathrm{v} \%$ ) and green (and poly[tris(2,5-bis(hexyloxy)-1,4-henylenevinylene)alt-(1,3-phenylenevinylene)] (PTDPV), $0.05 \mathrm{w} / \mathrm{v} \%$ ) fluorescent dyes were loaded into the two jetting solutions. The solutions were processed with a flow rate of $0.30 \mathrm{~mL} / \mathrm{h}$ for each flow. Meanwhile, a DC voltage between 7.5 and $9.0 \mathrm{kV}$ was applied to the capillaries, resulting in the formation of a single Taylor cone from the tip of a liquid droplet at the end of the capillaries. The subsequent formation of an electrified jet ensured rapid and controlled evaporation of volatile solvents and precipitation of the nonvolatile PLGA and TTBO components in form of spherical nanoparticles with Janus architectures. Scheme 1B summarizes the subsequent conversion of the composite particles into inorganic Janus catalysts that are capable of supporting selective growth of carbon nanotubes (CNTs) on one side of the particles. Calcination is carried out to obtain a more robust particle structure and, at the same time, to remove the sacrificial polymer template, resulting in pure inorganic Janus particles (here, $\mathrm{TiO}_{2}$ particles). The resulting particles are finally subjected to anisotropic catalytic growth of CNTs using a conventional chemical vapor deposition (CVD) process with $\mathrm{C}_{2} \mathrm{H}_{2}$ as the carbon source (see experimental section for further details).

Figure 1A presents a CLSM image of Janus PLGA/ titania composite particles collected immediately after EHD co-jetting. The clearly distinguishable interface between the viscosity increases, the jetting process becomes increasingly uncontrollable, and it is extremely difficult to create shapes, other than continuous fibers. ${ }^{[20]}$ In contrast to the reactive jetting used in the past, the jetting conditions employed in this work allow for substantially lower solution viscosities. Specifically, the use of anhydrous solvents and the absence of either acid or base catalysts favor the preparation of spherical particles. In addition, the jetting solutions are stable and do not significantly change in terms of viscosity or chemical composition of the solution during the time of jetting. As a result, jetting is stable for several hours or longer. Finally, this method provides the potential for incorporating inorganic nanocrystals into the Janus catalysts without altering the desired spherical morphology because the iron oxide is not subject to acid or base degradation.

The scanning electron micorscopy (SEM) image of the PLGA/TTBO composite particles shown in Figure 1B suggests that the particles have spherical shapes with an average diameter of $800 \pm 200 \mathrm{~nm}$, which is in good agreement with the dynamic light scattering (DLS) measurements (Figure 2A). The high-resolution SEM image in Figure 1C further reveals successful encapsulation of magnetite nanocrystals in only one compartment, which is associated with a rougher surface as compared to the porous surface of the second compartment, which does not contain magnetite. Figure 1D is an enlarged SEM image of the interface of two compartments. Several exposed magnetic nanoparticles can be observed on one side of these Janus particles (arrows). The iron oxide nanocrystals incorporated in one hemisphere remained chemically intact due to the fact that neither acids nor bases were used as a part of the jetting solutions. In addition, the localization of magnetite in only one compartment was observed by 2D energy dispersive X-ray spectroscopy (EDX) elemental tributions are shown in Figures $1 \mathrm{E}$ and indicate successful compartmentalization. A clear interface between the two compartments can be observed in the $\mathrm{Fe} / \mathrm{Ti}$ overlay image suggesting a bicompartmental composition of the PLGA/ TTBO composite particles. In addition, Figure $1 \mathrm{~F}$ presents the histogram of elemental distributions of $\mathrm{Ti}$ and $\mathrm{Fe}$ for the distribution mapping. The EDX maps for the Fe and Ti dis- 

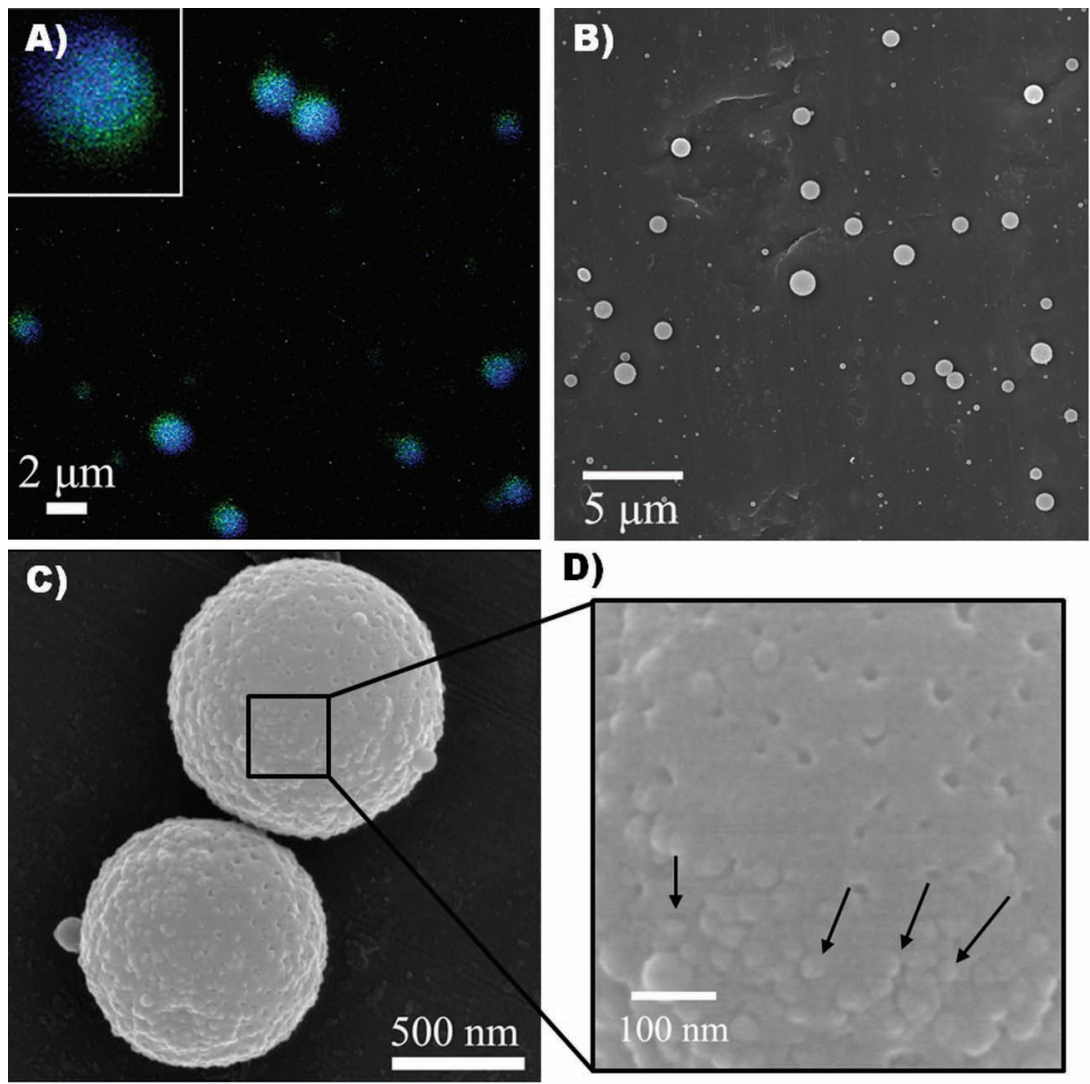

D)
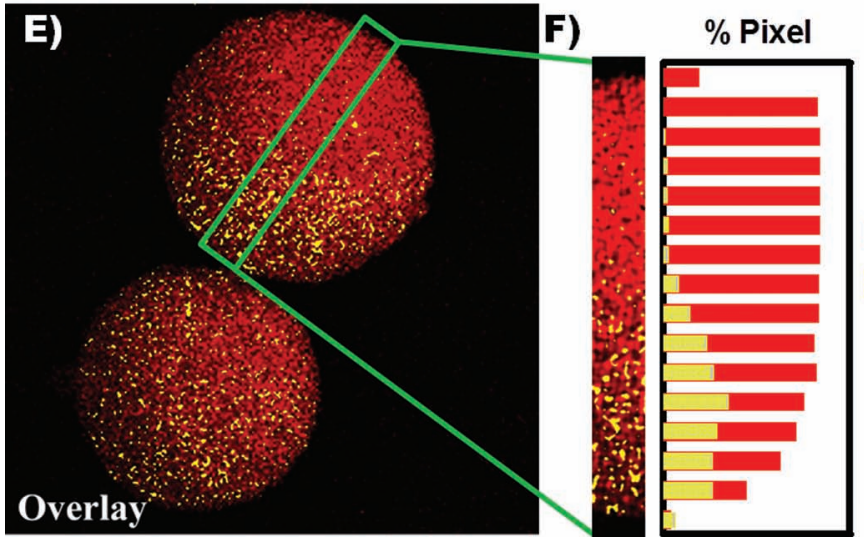

Figure 1. A) CLSM image of bicompartmental PLGA/TTBO composite particles, which are selectively loaded with MEHPV (blue) and PTDPV (green) dyes; inset shows a high-resolution image of a particle. B,C) Low- and high-magnification SEM images of the as-jetted spherical particles. D) Higher-magnification SEM image of (C), indicating that one face of the Janus particles are decorated with magnetic nanoparticles (arrows) with an average diameter of $30 \mathrm{~nm}$. E) 2D EDX images showing Fe (yellow) and Ti (red) distributions on the particles (the EDX image was obtained from C). F) Histogram displaying element distributions of a selected area of (E).

selected area shown in Figure 1E, revealing the compartmentalization of the magnetic nanoparticles within the Janus particles. Because of the simultaneous jetting of inorganic and organic components, which display large differences in their physical properties, the establishment and maintenance of a stable Taylor cone and jet during EHD co-jetting can be challenging. ${ }^{[21-24]}$ In fact, a number of control experiments were necessary to determine the 10:15:1 ratio of polymer, TTBO precursor, and iron oxide nanocrystals that is appropriate for the successful preparation of well-defined bicompartmental nanoparticles.

Titania precursors, such as TTBO, have high reactivity towards traces of environmental water. ${ }^{[28]}$ Because of the extremely large surface areas encountered during electrohydrodynamic co-jetting, there is a legitimate concern that the TTBO solutions may already be hydrolyzed during jetting by trace amounts of water in the atmosphere. This effect could potentially undermine the use of anhydrous solvents intended to prevent reactive jetting as described above. We thus jetted polymer/TTBO solutions and subsequently exposed the composite particles to anhydrous chloroform to dissolve the polymer matrix. Under these conditions, only small, scattered fragments of titania with sizes of a few tens of nanometers were observed in SEM analysis. This result suggests that the extent of chemical condensation of the titanium precursors is very limited only yielding ultra-small titania grains in the PLGA matrix.

One viable pathway towards fully condensed titania particles from as-jetted composite particles is the use of thermal calcination, which offers the benefit of producing the titania particles and removing the degradable polymer matrix in one single step. Accordingly, the as-jetted PLGA/TTBO composite particles were left in air for $2 \mathrm{~h}$ and then subjected to calcination at $500{ }^{\circ} \mathrm{C}$ for $4 \mathrm{~h}$ in air, resulting in condensed titania particles (Scheme 1B). Thermogravimetric analysis (TGA) reveals complete removal of PLGA after calcination (Figure S2, Supporting Information (SI)). As determined by DLS measurements in water (Figure 2A), the average hydrodynamic diameter of these bicompartmental particles decreased to $602 \pm 200 \mathrm{~nm}$ after calcination. This is consistent with the shrinkage of particles during condensation and thermal treatment. ${ }^{[29]}$ Assuming the density of PLGA and titania to be 1.2 and $4 \mathrm{~g} / \mathrm{cm}^{3}$, respectively, we estimated an average diameter reduction of $25 \%$, which is in good agreement with the results of the TGA analysis.

Before calcination, X-ray diffraction (XRD) patterns suggest the presence of amorphous $\mathrm{TiO}_{2}$. Several weak signals can further be attributed to $\mathrm{Fe}_{3} \mathrm{O}_{4}$ nanocrystals (black in Figure 2B). ${ }^{[30]}$ For comparison, the XRD spectra of the Janus catalysts after calcination are shown with/without iron oxide nanocrystals in one compartment. After calcination at $500{ }^{\circ} \mathrm{C}$ under ambient atmosphere, the titania particles took on a crystalline anatase phase, which is consistent with previously 

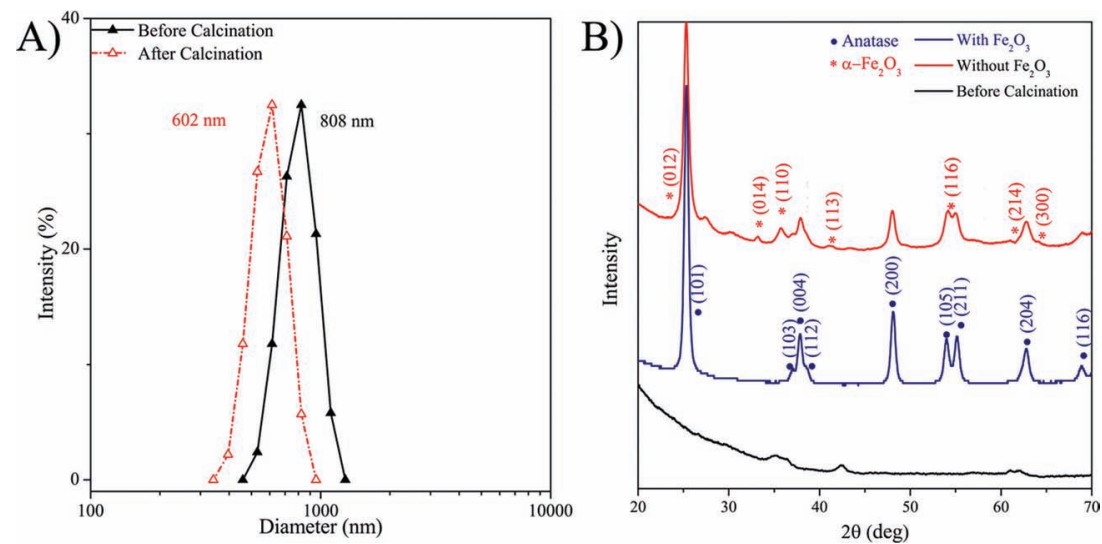

Figure 2. A) Typical DLS size distributions of bicompartmental particles before (black) and after (red) calcination, showing the decrease of mean hydrodynamic diameter from 808 to $602 \mathrm{~nm}$ after PLGA removal and $\mathrm{TiO}_{2}$ condensation by calculation. B) XRD patterns of the calcinated $\left(500{ }^{\circ} \mathrm{C}, 4 \mathrm{~h}\right) \mathrm{TiO}_{2}$ Janus catalysts with (blue) and without (red) $\mathrm{Fe}_{2} \mathrm{O}_{3}$, indicating the formation of anatase $\mathrm{TiO}_{2}$ and $\mathrm{Fe}_{2} \mathrm{O}_{3}$ after calcination (black: XRD patterns of the particles before calcination).

reported work. ${ }^{[31,32]}$ In addition, XRD analysis suggests conversion of the magnetite nanocrystals $\left(\mathrm{Fe}_{3} \mathrm{O}_{4}\right)$ to hematite $\left(\alpha-\mathrm{Fe}_{2} \mathrm{O}_{3}\right)^{[33]}$ Although the main role of the titania in the present work was that of a catalyst support, it is important to note that the anatase titania could potentially impart additional functionality to the particles in form of photocatalytic activity. ${ }^{[34,35]}$

Electron microscopy confirmed that the original spherical shape was maintained after calcination (Figures 3). Based on analysis of the SEM images, the average particle diameter of the Janus catalysts was $600 \pm 300 \mathrm{~nm}$, which was in good accordance with the DLS results shown in Figure 2A. After calcination, relatively uniform pores were observed on the particles' surface, which might be attributed to the removal of PLGA at the elevated temperatures of the calcination reaction. The surface pores on the catalytic supporter could be a potential advantage in terms of reactant accessibility to the catalytically active iron. Furthermore, the pore size can be tuned by either changing the titania precursor concentration or adjusting the calcination temperature (Figure S3, SI). Finally, transmission electron microscopy (TEM) imaging and 2D EDX analysis further supports the bicompartmental character of the Janus catalysts after calcination. Figure 3C and 3D shows a TEM image of bicompartmental Janus catalyst particles. The Janus nanoparticles display a contrast in Figure $3 \mathrm{C}$. This supports our assumption that the iron oxide nanocrystals are selectively confined to only one hemisphere of the Janus catalyst, and maintained their original shape and size (arrow in Figure 3D).
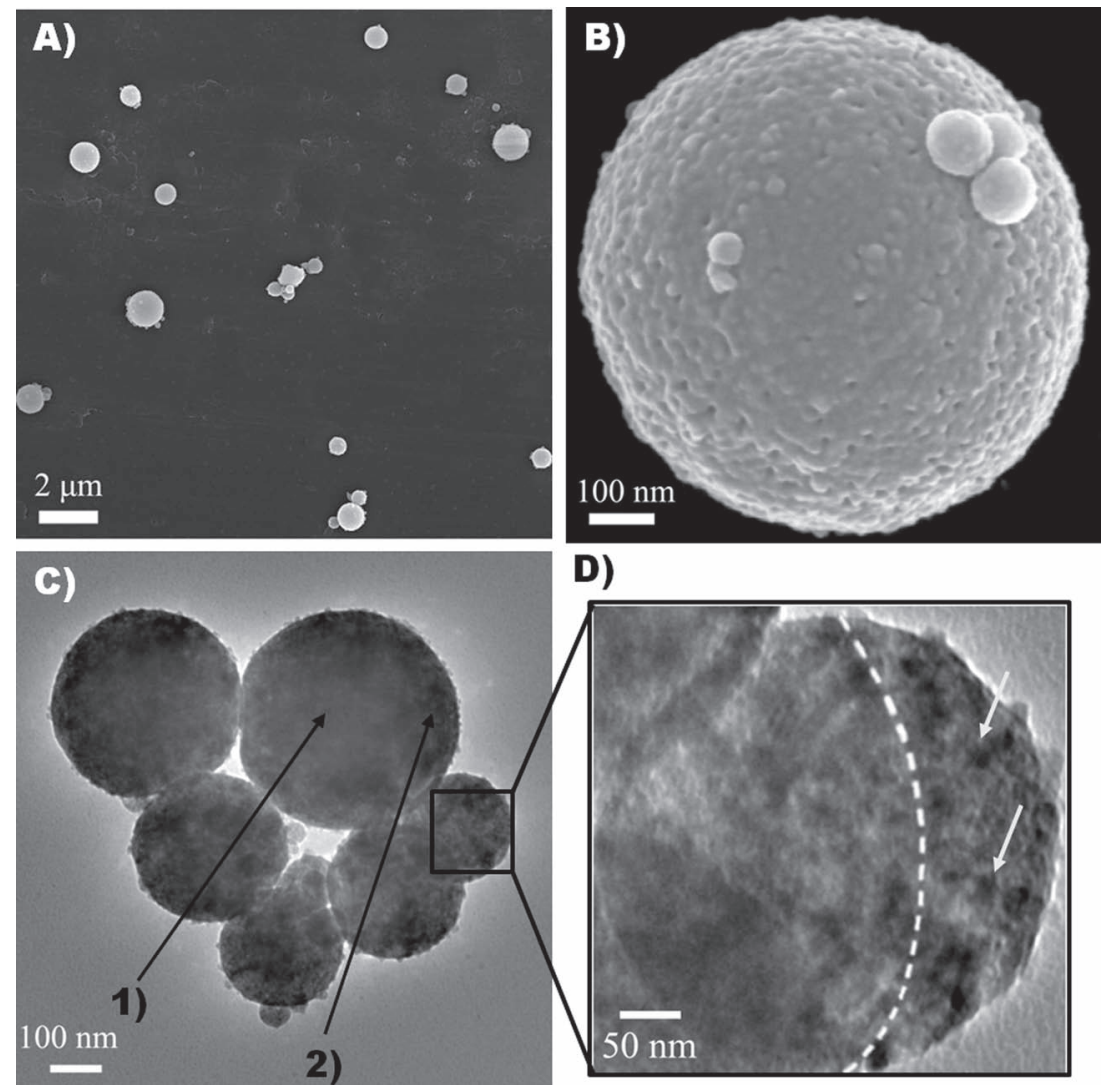

Figure 3. A, B) SEM images of Janus catalysts after calcination of PLGA/TTBO composite particles. C) TEM image of Janus catalysts supporter after calcination. Contrast differences between $\mathbf{1}$ (without magnetic particles) and $\mathbf{2}$ (with magnetic particles) can be depicted. D) Higher-magnification TEM image. Magnetic nanoparticles can be observed. 


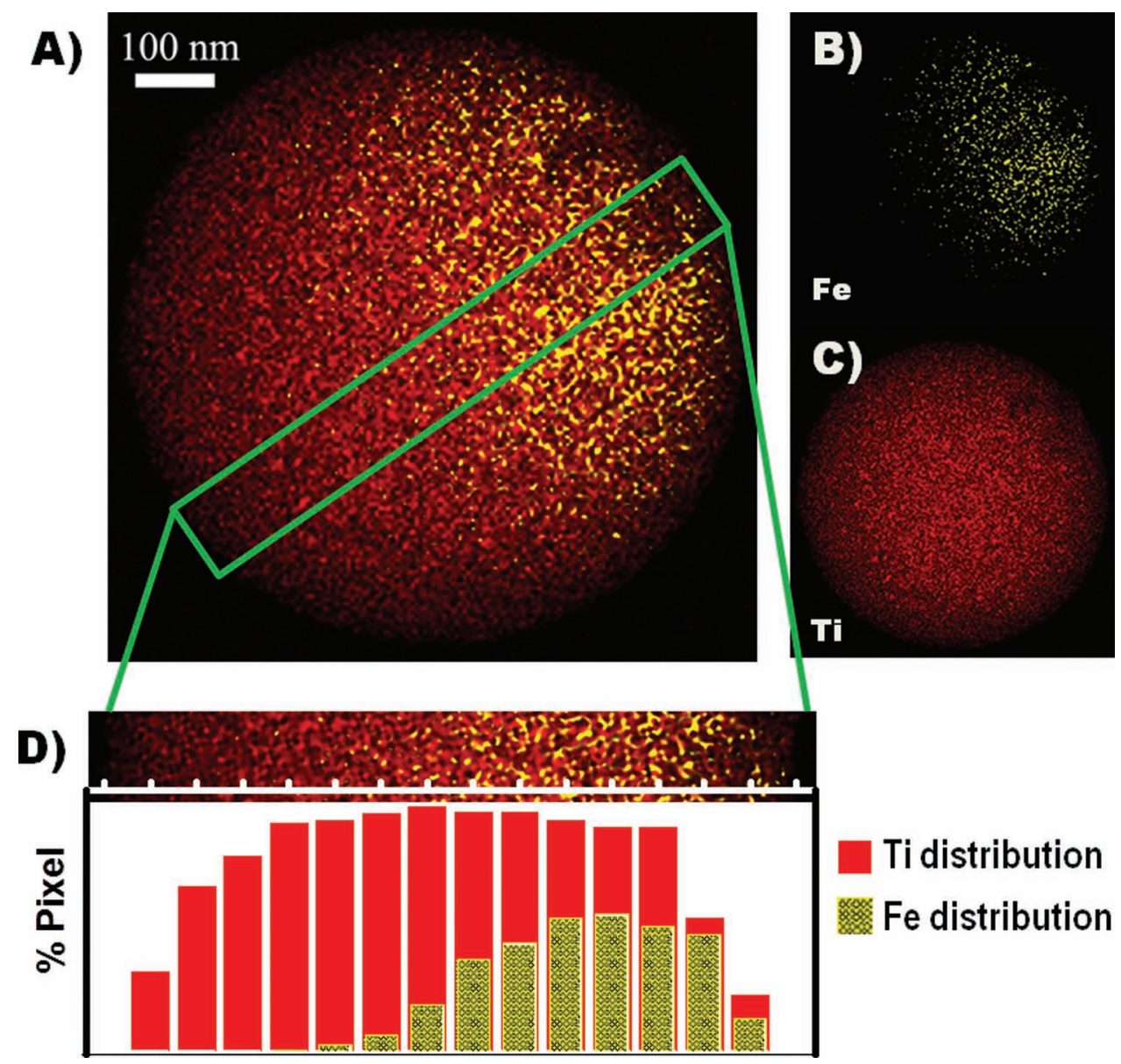

Figure 4. A) 2D elemental distribution map of the central region of a Janus particle. EDX mappings of the individual chemical elements are also displayed: B) Fe, C) Ti. D) Histogram displaying elemental distributions of a selected area.

controlled catalytic reaction using the Janus catalysts. In this case, we designed Janus catalyst particles for patterned catalytic growth of multiwalled CNTs (MWCNTs) from one hemisphere of the bicompartmental titania particles. Janus catalyst particles with iron nanocrystals localized in one compartment were placed on a quartz substrate and CNT growth was performed in a single-zone atmospheric pressure quartz tube furnace. After purging, the furnace temperature was increased from room temperature to $775^{\circ} \mathrm{C}$ within $10 \mathrm{~min}$, and held at $775{ }^{\circ} \mathrm{C}$ for another $10 \mathrm{~min}$, while flows of $400 \mathrm{sccm}$ (standard cubic centimeters per minute) $\mathrm{H}_{2}$ and $100 \mathrm{sccm}$ He were maintained. Under these conditions, iron oxide was reduced to metallic iron, which acted as the active sites for the growth of CNT. Finally, $\mathrm{C}_{2} \mathrm{H}_{2}$ was introduced into the furnace as the carbon source to grow the CNTs (see Experimental Section). ${ }^{[36]}$

As shown in Figure 5A, the growth of CNTs was indeed restricted to the hemisphere that contained the iron oxide nanocrystals. Moreover, the spherical shape of the Janus catalysts was fully maintained during the CNT growth in spite of the volatile high-temperature environment. The Raman spectrum of the "fuzzy" Janus particle displays the typical Raman shifts for MWCNTs (Figure S4, SI). ${ }^{[37]}$ In addition, Figure 5B shows a TEM image of MWCNTs grown on the Janus catalyst nanoparticles. Figure 5B also reveals that the MWCNTs encapsulate metal particles with diameters ranging between 20 and
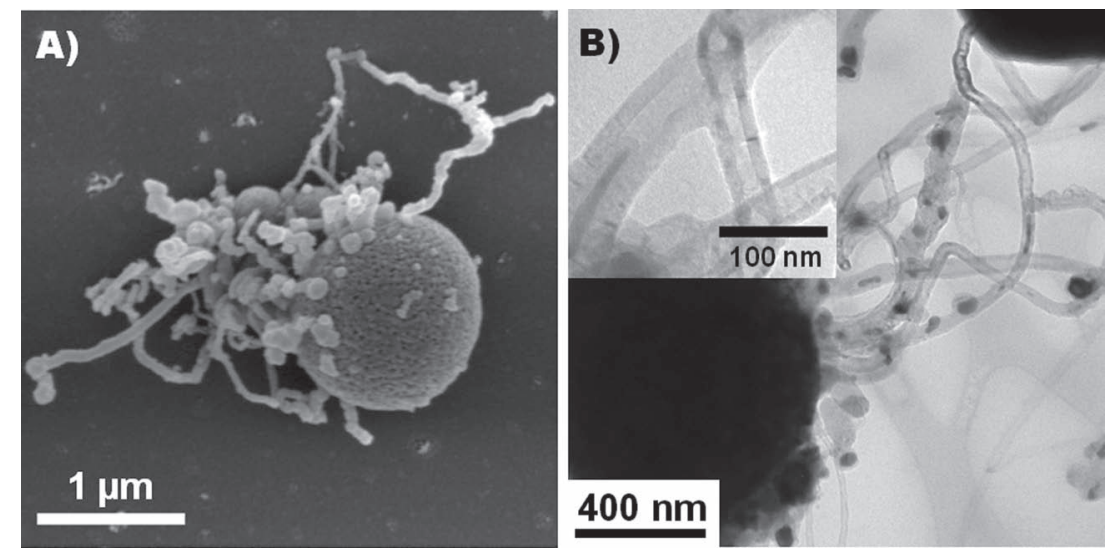

Figure 5. A) SEM and B) TEM images (inset: higher-magnification image) of anisotropic catalytic growth of CNTs on $\mathrm{TiO}_{2}$ particles using $\mathrm{C}_{2} \mathrm{H}_{2}$ as the carbon source. 
$40 \mathrm{~nm}$, which appeared to emerge from the surface of the spherical titania support. We suspect that the porosity of the titania particles allows the CNT precursors to penetrate the particle and enable protrusion of the CNTs from the particle surface, which would imply a strong physical attachment of the CNTs to the Janus catalyst. Future efforts to engineer the size and polydispersity of the metal particles within the titania spheres may provide opportunities for tuning the length, density, and alignment of the spatially controlled CNT coatings.

In this work, inorganic Janus nanoparticles were successfully produced through EHD co-jetting with anhydrous jetting solutions, taking advantage of a degradable templating polymer. The composite particles were subsequently converted into spherical Janus catalysts using thermal calcination. Importantly, the introduction of catalytic nanocrystals into one of the jetting solutions enabled the localization of catalyst into only one hemisphere. Finally, MWCNTs were grown from one hemisphere of the Janus catalysts in a spatially controlled manner. The herein described method is rather versatile and flexible and can be extended to produce a wide range of heterogeneous catalyst systems as well as catalytic supporters. This work thus broadens the available design space for particle engineers. ${ }^{[38]}$ Ultimately, these or similar particles may be instrumental in enabling heterogeneous multi-cascade reactions for synthetic chemists. Further, assembly of these particles into films and solids may provide a scalable means of using these custom catalyst systems for energy conversion applications. ${ }^{[14,15,39,40]}$

\section{Experimental Section}

Materials: All chemical reagents including PLGA (ratio of lactide and glycolide $=85: 15$; molecular weight, $M w=50-75000 \mathrm{kDa}$ ), TTBO, chloroform, and DMF were purchased from Sigma-Aldrich, USA and used without purification. MEHPV and PTDPV, which are used as visualizing markers for blue and green emission in CLSM, were purchased from Sigma-Aldrich, USA. Magnetic nanoparticles $(10 \mathrm{~nm})$ dispersed in chloroform were purchased from Ocean Nanotech, USA. Gases used in CVD process include He (Helium Uhp Gr 5.0 Size 300 CGA 580, Airgas), $\mathrm{C}_{2} \mathrm{H}_{2}$ (Acetylene AA Grade 2.8 Size Bulk Atomic Absorption Grade Airgas), $\mathrm{CO}_{2}$ (Carbon Dioxide Research Grade 5.0 Size 200 CGA 320, Airgas), and $\mathrm{H}_{2}$ (Hydrogen Uhp Gr 5 Size 300 CGA 350, Airgas).

Preparation of PLGA/TTBO Particles by EHD Co-jetting with Side-by-Side Dual Capillaries: The experimental set-up for EHD co-jetting of the composite particles is similar to our previous work. ${ }^{[21-24]}$ Two jetting solutions, which contain both $6.0 \mathrm{w} / \mathrm{v} \%$ PLGA and $9.0 \mathrm{w} / \mathrm{v} \%$ TतBO are prepared in organic solvent mixture of $\mathrm{CHCl}_{3}$ and DMF (97:3, v/v). One solution is loaded with trace amount of MEHPV, and the other with PTDPV and iron oxide nanoparticles. The final concentration of iron oxide nanoparticles is $0.6 \mathrm{w} / \mathrm{v} \%$. After gently shaking overnight, the jetting solutions are transferred to two syringes ( $1 \mathrm{~mL}$, Becton-Dickinson) and are ready for EHD co-jetting. The syringes are held together using a plastic syringe holder and connected with side-by-side dual capillaries (26 G, Hamilton Company, USA). The cathode of a DC voltage source (Gamma High Voltage Research, USA) is connected to the capillaries, and the counterelectrode is connected with a collecting aluminum (Al) foil or stainless steel plate for harvesting the bicomparmental composite particles. The distance between jetting capillaries and the collecting substrate is set to $\sim 25-30 \mathrm{~cm}$. The two solutions are pumped at the same time and the flow rate of each solution is maintained around $0.30 \mathrm{~mL} / \mathrm{h}$ by a syringe pump when a driving voltage around 7.5-9.0 kV is applied to the capillaries.

Preparation of Janus Catalysts by Calcination Reaction: The asjetted PLGA/TTBO composite particles are collected on top of the stainless steel substrate and transferred onto a quartz substrate. Next, they are calcinated to achieve removal of the templating polymer PLGA and polycondensation of TTBO, yielding bicompartmental $\mathrm{TiO}_{2}$ particles. The calcination temperature is controlled at $500{ }^{\circ} \mathrm{C}$ to generate controlled crystalline phases of the $\mathrm{TiO}_{2}$ particles. The Janus catalysts are suspended in $2 \% \mathrm{v} / \mathrm{v}$ Tween-20 water solution for further characterization and CNT growth.

Anisotropic Catalytic Growth of CNTs on Janus Catalysts by CVD: About $10 \mu \mathrm{L}$ of Janus catalysts suspended in water are casted on a small piece of quartz substrate $\left(\sim 1 \mathrm{~cm}^{2}\right)$, and water is allowed to evaporate at room temperature. CNT growth occurs in a single-zone atmospheric pressure quartz tube furnace (Lindberg) with a $30 \mathrm{~cm}$ long heating zone, with quartz tube of $22 \mathrm{~mm}$ inside diameter. The quartz tube is purged with $1000 \mathrm{sccm}$ He at room temperature for $5 \mathrm{~min}$, and followed by a combination of $400 \mathrm{sccm}$ of $\mathrm{H}_{2}$ and $100 \mathrm{sccm}$ of He for another $5 \mathrm{~min}$. Then the furnace is ramped up from room temperature to $775^{\circ} \mathrm{C}$ for $10 \mathrm{~min}$, and held at $775^{\circ} \mathrm{C}$ for another $10 \mathrm{~min}$, while flow is maintained at $400 \mathrm{sccm} \mathrm{H}_{2}$ and $100 \mathrm{sccm} \mathrm{He}$. Then the furnace temperature is retained at $775^{\circ} \mathrm{C}$ for $15 \mathrm{~min}$ under gas flow of $560 \mathrm{sccm} \mathrm{He}, 20 \mathrm{sccm} \mathrm{C} \mathrm{H}_{2}$, and $20 \mathrm{sccm}$ $\mathrm{CO}_{2}$. After the desired growth time elapses, the heat is turned off and the cover of furnace is opened, with a flow of $1000 \mathrm{sccm}$ of He until the quartz tube cools down to room temperature.

\section{Supporting Information}

Supporting Information is available from the Wiley Online Library or from the author.

\section{Acknowledgements}

The author thanks the American Cancer Society (RSG-08-28401-CDD) for financial support and acknowledges funding from the Multidisciplinary University Research Initiative of the Department of Defense and the Army Research Office (W911NF-10-1-0518). W. Lv acknowledges support from China Scholarship Council. KJ Lee was also supported by National Research Foundation of Korea Grant funded by the Korean Government (Ministry of Education, Science and Technology. (NRF - 2011 - 357 - D00055)

[1] Q. Chen, J. K. Whitmer, S. Jiang, S. C. Bae, E. Luijten, S. Granick, Science 2011, 331, 199-202.

[2] K. J. Lee, J. Yoon, J. Lahann, Curr. Opin. Colloid Interface Sci. 2011, 16, 195-202.

[3] J. Du, R. K. O'Reilly, Chem. Soc. Rev. 2011, 40, 2402-2416.

[4] S.-M. Yang, S.-H. Kim, J.-M. Lim, G.-R. Yi, J. Mater. Chem. 2008, $18,2177-2190$ 
[5] S. C. Glotzer, M. J. Solomon, Nat. Mater. 2007, 6, 557-562.

[6] S. Mitragotri, J. Lahann, Nat. Mater. 2009, 8, 15-23.

[7] J. Yoon, K. J. Lee, J. Lahann, J. Mater. Chem. 2011, 21, 8502-8510.

[8] V. N. Manoharan, M. T. Elsesser, D. J. Pine, Science 2003, 301, 483-487.

[9] D. Dendukuri, P. S. Doyle, Adv. Mater. 2009, 21, 4071-4086.

[10] R. K. Shah, J.-W. Kim, D. A. Weitz, Adv. Mater. 2009, 21, 1949-1953.

[11] Z. Li, E. Kesselman, Y. Talmon, M. A. Hillmyer, T. P. Lodge, Science 2004, 306, 98-101.

[12] S. Crossley, J. Faria, M. Shen, D. E. Resasco, Science 2010, 327, 68-72.

[13] C. Yu, Y. Zhang, A. Song, Y. Ji, W. Wang, Chem. Eur. J. 2011, 17, 770-774.

[14] G. A. Ozin, I. Manners, S. Fournier-Bidoz, A. Arsenault, Adv. Mater. 2005, 17, 3011-3018.

[15] a) P. M. Wheat, N. A. Marine, J. L. Moran, J. D. Posner, Langmuir 2010, 26, 13052-13055; b) L. Baraban, D. Makarov, R. Streubel, I. Mönch, D. Grimm, S. Sanchez, O. G. Schmid, ACS Nano 2012, DOI: 10.1021/nn300413p; c) S. Sanchez, A. A. Solovev, S. M. Harazim, O. G. Schmidt, J. Am. Chem. Soc. 2011, 133, 701-703; d) A. A. Solovev, E. J. Smith, C. C. B. Bufon, S. Sanchez, O. G. Schmidt, Angew. Chem. Int. Ed. 2011, 50, 10875-10878.

[16] S. Guo, S. Zhang, X. Sun, S. Sun, J. Am. Chem. Soc. 2011, 133, 15354-15357.

[17] D. Sun, V. Mazumder, O. Metin, S. Sun, ACS Nano 2011, 5, 6458-6464.

[18] A. Greiner, J. H. Wendorff, Angew. Chem. Int. Ed. 2007, 46, 5670-5703.

[19] X. Lu, C. Wang, Y. Wei, Small 2009, 5, 2349-2370.

[20] J. Di, Y. Zhao, J. Yu, J. Mater. Chem. 2011, 21, 8511-8520.

[21] S. Bhaskar, J. Hitt, S.-W. L. Chang, J. Lahann, Angew. Chem. Int. Ed. 2009, 48, 4589-4593.

[22] K. H. Roh, D. C. Martin, J. Lahann, Nat. Mater. 2005, 4, 759-763.

[23] K. H. Roh, D. C. Martin, J. Lahann, J. Am. Chem. Soc. 2006, 128, 6796-6797.

[24] S. Bhaskar, K. M. Pollock, M. Yoshida, J. Lahann, Small 2010, 6, 404-411.
[25] S. Zhan, D. Chen, X. Jiao, Y. Song, Chem. Commun. 2007, 2043-2045.

[26] E. Formo, E. Lee, D. Campbell, Y. Xia, Nano Lett. 2008, 8, 668-672.

[27] Z. Liu, D. D. Sun, P. Guo, J. O. Leckie, Nano Lett. 2007, 7, 1081-1085.

[28] J. Stotzel, D. Lutzenkirchen-Hecht, R. Frahm, C. V. Santilli, S. H. Pulcinelli, R. Kaminski, E. Fonda, F. Villain, V. Briois, J. Phys. Chem. C 2010, 114, 6228-6236.

[29] M. Koneracka, V. Zavisova, M. Timko, P. Kopcansky, N. Tomasovicova, K. Csach, Acta Phys. Polonica A 2008, 113, 595-598.

[30] S. Sun, H. Zeng, D. B. Robinson, S. Raoux, P. M. Rice, S. X. Wang, G. Li, J. Am. Chem. Soc. 2003, 126, 273-279.

[31] D. Li, Y. Xia, Nano Lett. 2003, 3, 555-560.

[32] P. Kubiak, T. Fröschl, N. Hüsing, U. Hörmann, U. Kaiser, R. Schiller, C. K. Weiss, K. Landfester, M. Wohlfahrt-Mehrens, Small 2011, 7, 1690-1696.

[33] a) M. Cao, T. Liu, S. Gao, G. Sun, X. Wu, C. Hu, Z. L. Wang, Angew. Chem. Int. Ed. 2005, 44, 4197-4201; b) X. Yang, L. Li, Nanotechnology 2010, 21, 355602.

[34] J. M. Macak, M. Zlamal, J. Krysa, P. Schmuki, Small 2007, 3, 300-304.

[35] G. Li, J. Liu, G. Jiang, Chem. Commun. 2011, 47, 7443-7445.

[36] A. Magrez, J. W. Seo, R. Smajda, B. Korbely, J. C. Andresen, M. Mionic, S. Casimirius, L. Forro, L. ACS Nano 2010, 4, 3702-3708.

[37] J. Yang, Y. Yang, S. W. Waltermire, T. Gutu, A. A. Zinn, T. T. Xu, Y. Chen, D. Li, Small 2011, 7, 2334-2340.

[38] J. Lahann, Small 2011, 7, 1149-1156.

[39] S. Cha, S. M. Kim, H. Kim, J. Ku, J. I. Shon, Y. J. Park, B. G. Song, M. H. Jung, E. K. Lee, B. L. Choi, J. J. Park, Z. L. Wang, J. M. Kim, K. Kim, Nano Lett. 2011, 11, 5142-5147.

[40] A. Kumar, H. Gullapalli, K. Balakrishnan, A. Botello-Mendez, R. Vajtai, M. Terrones, P. M. Ajayan, Small 2011, 7, 2173-2178.

Received: January 26, 2012

Revised: May 15, 2012

Published online: August 3, 2012 